This item was submitted to Loughborough's Research Repository by the author.

Items in Figshare are protected by copyright, with all rights reserved, unless otherwise indicated.

\title{
Increased physical activity improves sleep and mood outcomes in inactive people with insomnia: a randomized controlled trial
}

PLEASE CITE THE PUBLISHED VERSION

http://dx.doi.org/10.1111/jsr.12297

\section{PUBLISHER}

Wiley ( European Sleep Research Society

\section{VERSION}

AM (Accepted Manuscript)

\section{PUBLISHER STATEMENT}

This work is made available according to the conditions of the Creative Commons Attribution-NonCommercialNoDerivatives 4.0 International (CC BY-NC-ND 4.0) licence. Full details of this licence are available at: https://creativecommons.org/licenses/by-nc-nd/4.0/

\section{LICENCE}

CC BY-NC-ND 4.0

\section{REPOSITORY RECORD}

Hartescu, Iuliana, Kevin Morgan, and Clare Stevinson. 2015. "Increased Physical Activity Improves Sleep and Mood Outcomes in Inactive People with Insomnia: A Randomized Controlled Trial”. Loughborough University. https://hdl.handle.net/2134/19679. 


\section{INTRODUCTION}

Recognized as an important public health issue (Altevogt and Colten, 2006), insomnia is estimated to affect approximately $10 \%$ of the adult population (Morin et al., 2006) and is associated with chronic symptoms of fatigue (Orff et al., 2007), impairment of sustained attention (Altena et al., 2008), poorer working memory (Bonnet and Arand, 1995) and degraded quality of life (Bolge et al., 2009). Regular exercise has been associated with a range of public health benefits, which include a reduced risk of coronary heart disease (Sattelmair et al., 2011), diabetes (Jeon et al., 2007), cancers (Winzer et al., 2011) and neurodegenerative disorders (Hamer and Chida, 2009). The evidence also suggests causal and therapeutic linkages between exercise and sleep quality (Youngstedt, 2005).

While earlier studies tended to focus on sleep outcomes following relatively high intensity activities and athletic performance (see (Driver and Taylor, 2000)), recent clinical trial findings have demonstrated moderate, but significant sleep benefits for lower intensity exercise (for a recent systematic review, see (Yang et al., 2012)). Programs requiring 100 minutes (Reid et al., 2010) to 210 minutes (King et al., 2008) of weekly activity, delivered over periods ranging from 16 weeks (King et al., 1997) to 54 weeks (King et al., 2008) have shown significant improvements in sleep quality among those with "mild” to "moderate" sleep complaints (Irwin et al., 2008); or “chronic” insomnia symptoms (Li et al., 2004, Reid et al., 2010). These physical activity programs included walking and jogging, generic endurance exercises, or tai chi.

As yet, however, there is little evidence supporting an optimal type or minimal level of physical activity which can positively impact sleep quality, and provide clear guidance for public health or clinical interventions for insomnia. Given the clinical trials evidence to date, 
a logical candidate level of activity is that currently recommended by the World Health Organization (WHO, 2010) and embedded in cardiovascular health promotion advice throughout the world: 150 minutes per week of moderate intensity activity, usually taken as walking.

In addition to reflecting a range of activity types, intensities and program durations, the present clinical trials literature also lacks standardization in the definition of insomnia, and the selection of outcome measurements of sleep quality. Furthermore, none of the studies assessing the impact of moderate intensity activity on sleep outcomes has controlled for the possibly confounding effect of daylight exposure which inevitably increases as outdoor activity increases. Such exposure could, independent of exercise, influence sleep outcomes (Guilleminault et al., 1995, Gordijn et al., 1998, Youngstedt et al., 2002).

The present trial, therefore, was designed to test whether, in analyses controlling for light exposure, minimum physical activity to the level of the current public health guidelines (150 minutes of moderate intensity activity/week) can improve sleep quality among inactive individuals meeting research diagnostic criteria for insomnia (Edinger et al., 2004). Informed by international recommendations for insomnia assessment in research studies (Buysse et al., 2006), trial outcomes also included measurements of light exposure, mood, and fatigue.

\section{METHODS}

\section{Design}

The study was designed as a two-arm (activity intervention versus wait-list control) parallel randomized controlled trial. Assessments were conducted at baseline, and at 6 months post-baseline. The activity intervention lasted 6-months, commencing immediately 
after the baseline assessments, and ending at the post-baseline assessment. The study was approved by Loughborough University Ethical Advisory Committee.

\section{Participants}

Inclusion criteria were: men or women aged 40 years or older; meeting Research Diagnostic Criteria for insomnia (Edinger et al., 2004); ambulant and independent in activities of daily living; and participation in moderate to vigorous intensity physical activity had not exceeded 60 minutes in a week, over the previous 6 months. Exclusion criteria were: symptoms of excessive daytime sleepiness or restless legs syndrome (assessed using validated questionnaires); history of cognitive or neurological disorders; history of any major psychiatric disorder; significant depressive symptoms as indicated by Beck Depression Inventory II score >31; the presence of chronic disease for which moderate-intensity physical activity would be contraindicated; engaged in shift work or other self-imposed irregular sleep schedules; a body mass index (BMI) > 35; history of smoking over the previous 6 months; or caffeine consumption greater than 300 mg per day. For women, being pre- or perimenopausal and/or receiving oestrogen replacement therapy also served as exclusion criteria.

Participants were recruited through advertising in the local commercial press, radio stations, local government organizations, local community organizations, and local major employers.

\section{Procedure}

Following screening, using custom and standardized questionnaires and selfcompletion interviews, those eligible were invited to participate in the study. After providing informed consent participants were randomized to a moderate-intensity physical activity condition, or a wait-list control condition. The algorithm for randomization used the random 
permuted-block procedure (blocks of 4), and was stratified by gender (male/female) and age ( \pm 2 years in each group). The randomization was performed by an independent researcher who had no other involvement in the study.

Six participants withdrew or were excluded following randomization. The reasons given for withdrawal by 3 allocated to the control group were: that they wanted to be in the physical activity condition ( $n=2)$, and worsening health $(n=1)$. For the remaining 3 allocated to the intervention group, the reasons given for withdrawal were unexpected lifestyle demands making time commitment to the trial difficult $(n=1)$, and worsening health $(n=2)$.

Intervention

Intervention group

Participants were instructed to engage in moderate intensity physical activity as 'brisk walking', in their chosen setting, in their own time. The intervention commenced with a conditioning period of 4 weeks, after which participants were required to engage in walking on at least 30 minutes per day, on at least 5 days of the week, totaling at least 150 minutes of moderate intensity physical activity at the end of each week.

To monitor adherence to the condition assigned, intervention participants were asked to wear a NewLife NL-1000 activity monitor (New Lifestyles Inc., US) for the duration of the intervention (6 months). The NL-1000 is a small hip-worn pedometer with accelerometer, which displays both step counts, and the total time in minutes spent at or above the moderate to vigorous intensity physical activity level (MVPA), which were then recorded daily in a physical activity diary.

\section{$\underline{\text { Control group }}$}


Participants were asked to continue their lifestyle as usual, and maintain their baseline levels of physical activity. To monitor activity and control for the possible motivational effect of wearing a physical activity monitoring device, participants in the control group were randomly assigned to wear the NewLife NL-1000 activity monitor for a period of 3 consecutive months.

\section{Outcome measures}

Following baseline measurement, clinical outcomes were assessed at 6 months. Adherence was monitored throughout the trial. The primary outcome was the Insomnia Severity Index (ISI), a self-report questionnaire assessing the severity and impact of insomnia (Morin et al., 1993). The total score ranges from 0 to 28, with a score of 10 or above considered optimal (86.1\% sensitivity and $87.7 \%$ specificity) for detecting insomnia cases in community samples. In a clinical sample, change scores of -8.4 points $(95 \% \mathrm{CI}:-7.1,-9.4)$ have been associated with moderate improvement as rated by an independent clinical assessor after treatment (Morin et al., 2011).

Secondary outcomes included depression symptoms, assessed using the Beck Depression Inventory (BDI; Beck et al., 1988), while trait anxiety was measured using the Trait Anxiety Inventory (STAI; Spielberger et al., 1983). Additional measures were daytime sleepiness, assessed using the Epworth Sleepiness Scale (ESS; Johns, 1991), and daytime fatigue severity, assessed with the Fatigue Severity Scale (FSS; Krupp et al., 1989). Health related quality of life was assessed using the self-completed EuroQoL5D-5L (Herdman et al., 2011). BMI was calculated from self-reported height and weight measurements. 
Actigraphically measured sleep variables were derived from the Actiwatch 2 (Philips Respironics Inc, US), which was worn continuously for 14 days at baselines, and at 6 months (post-intervention). Sleep variables were calculated using the proprietary Respironics Actiware 5 (v.5.71, Phillips Electronics, US) software: total sleep time (TST), sleep onset latency (SOL), sleep efficiency (SE), wake after sleep onset (WASO), and sleep fragmentation index (SFI). The Actiwatch 2 incorporates a light sensor, which records daily photopic illuminance. Average daily light exposure (lux/min) was also calculated.

Physical activity was measured using actigraphy (GTX3+, Actigraph, US). Participants were instructed to wear the Actigraph around their waist, continuously during waking hours, for 14 days, at baseline, and then at 6 months. ActiLife software (v.6.5.3 Actigraph, US) was used for processing the data from GTX3+ accelerometers which, using published protocols (Troiano et al., 2008) to convert accelerometer data into MVPA minutes per week.

This multiple use of instrumental activity measures was considered necessary as the NL-100 delivered only adherence data. The more sophisticated research-grade actigraphic devices (Actiwatch 2 and the GTX3+ Actigraph) sample at different frequencies (32Hz v $30 \mathrm{~Hz}$ ), and use different equations to summarize movement. As a consequence, dual use was necessary to supply accurate and equation-specific measures of sleep (from the Actiwatch 2) and MVPA (from the GTX3+ Actigraph).

Analyses

Data were entered into SPSS version 20.0 (SPSS Statistics v. 20.0, IBM, US). Data was analyzed using pre-planned descriptive statistics, t-tests for independent means, analyses 
of variance and covariance, Pearson correlation and logistic regression. Data were checked for outliers, distribution variance, and missing values. Statistical assumptions were checked with normality tests. Statistical significance was defined as $\mathrm{p}<.05$ using two tailed tests, and Bonferroni corrections were used for between groups multiple comparisons.

All outcome scales were coded as continuous variables. Analyses of covariance (ANCOVA) models were employed, in which the dependent variable was the change prepost intervention (baseline minus 6 month follow-up) for each of the primary and secondary outcomes, and group membership (control or intervention) was a fixed factor. Assumptions of independence of the covariates and intervention effects, and homogeneity of regression slopes, were checked and met through a series of interaction univariate models, controlling for the main effects.

Logistic regression models were used to characterize the relationship between changes in scores on the Insomnia Severity Index (from baseline to 6 months postintervention) and outcomes from the Beck Depression Inventory, and the State-Trait Anxiety Inventory.

Effect sizes and confidence intervals were computed using the Effect Size Generator Professional Edition software (v. 4.1, ClinTools Software, Melbourne, Australia).

\section{Sample size calculation}

Sample size estimation was performed using GPower v.2 (Kiel University, Germany), and adopted the treatment effect size of physical activity on sleep quality in a community sample of people with sleep complaints found in previous randomized controlled trials(King 
et al., 1997) of 1.25 . Setting beta at $20 \%$, and alpha at $5 \%$, an allocation ratio of $1: 1$, it was found that a minimum of 12 participants per group was required in order to detect this effect size in the main outcome (ISI).

\section{Missing data}

A two stage process was used for handling missing data. In stage 1 separate bias analysis were conducted for data missing from participants who were randomized, but dropped out before the first follow up period. If stage 1 analysis revealed no bias effect of the missing data on the outcomes of the trial, then no data imputation would be performed. If the analyses at Stage 1 revealed that missing data biased the outcomes of the trial, then Stage 2 could be employed. In Stage 2, statistical procedures for generating missing values would have been used (multiple imputation or last observation carried forward methods). All data was analyzed on an intention to treat basis using SPSS version 20.0 (IBM, SPSS Statistics 20.0).

\section{RESULTS}

The full CONSORT diagram is presented in Figure 1. Participants’ sociodemographic, sleep and health characteristics at baseline are shown in Table 1. Briefly, the sample consisted of 41 adults (30 females), with a mean age of 60 years. The mean weekly volume of MVPA was 57 minutes. The sample mean BMI score was 26. There were no significant differences between the two groups at baseline on any of the variables of interest. None of the variables violated the equality of variance assumption, or normality of distributions assumption (for the t-tests). 
The depression and anxiety scores place the sample within the subclinical range. The mean ISI score placed this sample in the moderately severe range of insomnia severity. In terms of the development of the disorder, participants reported an average of 11 years of chronic persistence of insomnia, and $24 \%$ of participants reported present use of nonpharmacological and of pharmacological treatment to improve sleep. Participants reported on average having one chronic disease in addition to insomnia. Insomnia duration, medication use and number of other chronic diseases did not significantly differ between groups at baseline. Table 2 displays baseline sleep characteristics from the two weeks period of actigraphy monitoring before the intervention commenced. Again, there were no significant differences on any of these variables between the control and intervention groups.

\section{Missing data}

Logistic regression was employed to see whether key variables at baseline predicted 'missingness' of data at subsequent follow-up time of 6 months. Predictive relationships were examined between these adherence categories and key independent variables: group allocation, insomnia severity, health status, and mood. The analyses revealed no significant association between key variables and 'missingness' of data at 6 months. Since this outcome was consistent with data being missing at random, no imputation of missing values was performed.

\section{Adherence}

Over the trial, the participants in the intervention group engaged on average in 219 min/week ( $S D=99.75$ ) of moderate to vigorous intensity physical activity. The participants in the control group engaged on average in 74.38 minutes per week of MVPA (SD = 67.36). 
During the 6 month period of the intervention, the participants in the intervention group ( $\mathrm{N}=17)$ were aiming to reach at least 30 minutes of MVPA, over 5 days, per week, leading to at least 150 minutes of MVPA per week. When using this threshold to calculate regular adherence, out of 442 total reported weeks for the 17 participants, there were $4.75 \%$ weeks achieving less than 150 minutes MVPA per week. Thus, there was 95\% continuous adherence to the intervention. All the figures reported are from actigraphy activity monitoring.

\section{Main outcome}

There was a significant effect of group membership on change scores in ISI, after controlling for appropriate variables: $\mathrm{F}(8,26)=5.16, \mathrm{p}=.03$ (Table 3). Pre-post comparisons for each group showed a significant improvement in the ISI score in the physical activity group $(\mathrm{t}(16)=3.35, \mathrm{p}<0.01)$, but no significant change in the control group $(\mathrm{t}(17)=1.81, \mathrm{p}=0.21)$.

In order to determine the clinical significance of the change in ISI, a series of comparisons were performed within sample groups. The ISI was categorized as subthreshold insomnia (<14) and moderate to severe (15 and over) (Morin et al, 2011). Upon comparing the change in the two categories within the groups over time, there was a reduction of $67 \%$ in the moderate to severe category of the intervention group, compared to a $14 \%$ reduction within the same category in the control group (Figure 2). Overall, in the intervention group, there was an average 4-point reduction in the ISI score.

\section{Secondary outcomes}

ANCOVA models showed a significant effect of group membership on change in depression scores: $\mathrm{F}(6,28)=5.61, \mathrm{p}=0.02$. In pre-post comparisons for each group, participants 
in the physical activity group demonstrated significant improvement in the BDI scores, $\mathrm{t}(16)=4.03, \mathrm{p}<0.001$. Participants in the control group did not show significant improvement, $t(17)=-.61, p=0.54$. There was also a significant effect of group membership on trait anxiety outcomes post-intervention, $\mathrm{F}(6,28)=4.41, \mathrm{p}=0.05$. Follow-up comparisons for each group revealed that participants in the intervention group had significant improvement in their trait anxiety scores, whilst participants in the control group did not show the same pattern $(\mathrm{t}(16)=3.71, \mathrm{p}<0.01$, and $\mathrm{t}(17)=-.18, \mathrm{p}=0.34$, respectively).

There was no significant effect of group membership on the change score on Fatigue Severity Scale post-intervention, $F(8,26)=1.84, p=0.18$. Daytime sleepiness followed the same trend, with no significant effect of group membership on change scores at postintervention, $\mathrm{F}(8,26)=.04, \mathrm{p}=0.83$. The univariate models showed no significant effect of treatment condition on daily light exposure outcome post-intervention: $F(6,28)=.47, p=0.49$.

In a final exploratory analysis, a Pearson-product moment correlation was calculated between ISI scores and the Fatigue Severity Scale scores for the intervention group. Higher Insomnia Severity Index scores were associated with larger Fatigue Severity Scale scores ( $r=.58, \mathrm{p}=0.01$, 2-tailed). When analyzed as change scores (baseline - post-intervention), there was a significant positive association between changes in insomnia severity symptoms and changes in fatigue scores ( $\mathrm{r}=.50, \mathrm{p}=0.04,2$-tailed).

\section{DISCUSSION}

Achieving a minimum level of 150 minutes of moderate intensity physical activity, distributed over 5 days per week, is a public health aspiration recommended by the World Health Organization (WHO, 2010) and adopted by healthcare systems throughout the world. 
The present trial indicates that, among otherwise inactive individuals meeting research diagnostic criteria for insomnia, this level of activity is associated with significantly reduced insomnia symptoms severity, and significantly elevated mood. Importantly, these significant improvements in sleep and mood indicators were independent of average daily light exposure, as well as participants' health and social status.

Sleep outcomes showed a relatively large effect size (.78), with the intervention group showing an average 4-point reduction on the ISI scale at follow-up. On completion of the trial, $67 \%$ of the intervention group participants had moved into a lower ISI category. Given that the selection procedures used in the present study were designed to optimize external validity by recruiting a clinically heterogeneous sample, it is reasonable to conclude that, in relation to insomnia severity, the present study delivered modest, but clinically significant changes (see (Morin et al., 2011)). These results are consistent with a recent meta-analysis examining the effects of higher-level exercise interventions on sleep outcomes in middleaged and older people with sleep problems, which noted modest, but significant effects (Yang et al., 2012). It is therefore reasonable to conclude that lifestyle interventions, such as physical activity, should be considered in clinical practice as an effective therapy where appropriate, before other therapeutic measures which may have more detrimental side effects.

It has been suggested that the effects of exercise on sleep may be confounded by light exposure (Youngstedt et al., 2003), which, by itself, has chronobiotic properties (Guilleminault et al., 1995). However, no earlier study of the effects of a physical activity intervention on sleep outcomes has tested, or controlled for, increased exposure to light potentially arising from increased time spent outdoors. One of the strengths of the present trial, therefore, is the analysis of the effect of light exposure when interpreting the results of the intervention. While light exposure values did increase within the intervention group, this 
increase did not reach statistical significance, and did not significantly contribute to the relationship between physical activity and sleep outcomes in this sample. Thus, in interpreting the improvements resulting from the intervention, there appears to have been no confounding effect of increased light exposure.

The finding that the present physical activity intervention was associated with significant post-intervention reductions in depression and anxiety (as reflected in BDI and STAI scores) is consistent with both the physical activity, and the emerging physical activity and sleep literature. In a recent Cochrane review, Rimer and colleagues (2013) concluded that chronic exercise of at least one week duration can be effective in the treatment of depression. A recent sleep trial (Reid et al., 2010) showed significant reductions in depressive symptoms among sedentary adults with chronic insomnia following a 16 week program of aerobic activity. Physical activity interventions have previously shown a larger significant effect on trait anxiety (Herring et al., 2010), as was also found in the present study. Psychological mediational hypotheses to explain these findings include changes in self-efficacy, and the interruption of negative thoughts. These particular mechanisms have resonance in cognitive mechanisms by which insomnia may act (Harvey, 2002, Espie et al., 2006)

In the present trial, post-treatment comparisons of fatigue and daytime sleepiness scores showed no significant differences between groups. Nevertheless, additional analyses provided several lines of evidence indicating that changes in insomnia severity may have positively impacted fatigue experiences. It is interesting, therefore, that this pattern of results, indicative of significant change in daytime experience related to insomnia symptom severity, was not captured by the specific fatigue (FSS) measure employed. These results indicate that in people with insomnia, disturbed sleep may not be the only pathway leading to higher levels of fatigue, and that sleep and fatigue can vary independently. As a result, daytime fatigue 
experiences of people with insomnia may be best captured by a multidimensional approach, enquiring into the physical, cognitive and emotional domains (Dittner et al., 2004).

Several limitations of the present trial should be considered when interpreting these findings. It is not known at what point in the day or night participants took their exercise, as these measures were not analysed.. Given the multi-factorial nature of insomnia, light exposure might only (or mainly) affect those groups of people whose insomnia is related to circadian dysfunction, as it is the case with people with non-seasonal depression (Even et al., 2008). The menstrual cycle phase of female participants was not recorded through the trial, thus such independent effects on sleep quality are not known. The present trial employed an intervention testing the transition from inactivity, to activity levels to the current international guidelines, as this is the time point of most benefit. Current evidence also indicates a doseresponse relationship between physical activity and health outcomes, with increased doses of physical activity accompanied by increased significant improvements in health outcomes (Kline et al., 2012). It is also not known what the effects on sleep quality would be if existing levels of physical activity in adults already meeting the current guidelines would be increased even further. Future trials should help elucidate the physical activity and sleep quality dose response relationship.

The pattern of results reported here allow for two conclusions with clear implications for public health: 1) measures to increase levels of physical activity above the currently recommended threshold of 150 minutes per week could usefully be added to the other tools available for insomnia management; and 2) the likelihood of improved sleep quality should be routinely added to those evidence-based cardiovascular and metabolic benefits most frequently associated with increased physical activity in behavior change initiatives. 
Acknowledgements: The authors wish to thank the participants for their valuable contribution. 


\section{REFERENCES}

Altena, E., Van Der, W., Ysbrand, D., Strijers, R. L. M. and Van Someren, E. J. W. Sleep loss affects vigilance: Effects of chronic insomnia and sleep therapy. J.Sleep. Res., 2008, 17: 335-343.

Altevogt, B. M. and Colten, H. R. Sleep Disorders and Sleep Deprivation: An Unmet Public Health Problem. National Academies Press, London, 2006.

Beck, A. T., Steer, R. A. and Carbin, M. G. Psychometric properties of the Beck Depression Inventory: Twenty-five years of evaluation. Clin. Psychol. Rev., 1988, 8: 77-100.

Bolge, S. C., Doan, J. F., Kannan, H. and Baran, R. W. (2009). Association of insomnia with quality of life, work productivity, and activity impairment. Qual. Life. Res., 2009, 18: 415-422.

Bonnet, M. H. and Arand, D. L. 24-Hour metabolic rate in insomniacs and matched normal sleepers. Sleep, 1995, 18: 581-588.

Buysse, D. J., Ancoli-Israel, S., Edinger, J. D., Lichstein, K. L. and Morin, C. M. Recommendations for a standard research assessment of insomnia. Sleep, 2006, 29: 1155-1173.

Dittner, A. J., Wessely, S. C. and Brown, R. G. The assessment of fatigue: a practical guide for clinicians and researchers. J. Psychosom. Res., 2004, 56: 157-170.

Driver, H. S. and Taylor, S. R. Exercise and sleep. Sleep Med. Rev., 2000, 4: 387-402.

Edinger, J. D., Bonnet, M. H., Bootzin, R. R. et al. Derivation of research diagnostic criteria for insomnia: report of an American Academy of Sleep Medicine Work Group. Sleep, 2004, 27: 1567-1596.

Espie, C. A., Broomfield, N. M. and MacMahon, K. The attention-intention-effort pathway in the development of psychophysiologic insomnia: a theoretical review. Sleep Med. Rev., 2006, 10: 215-245. 
Even, C., Schröder, C. M., Friedman, S. and Rouillon, F. Efficacy of light therapy in nonseasonal depression: A systematic review. J. Affect. Disord., 2008, 108: 11-23.

Gordijn, M., Beersma, D., Korte, H. and Van Den Hoofdakker, R. Testing the hypothesis of a circadian phase disturbance underlying depressive mood in nonseasonal depression. $J$. Biol. Rhythms, 1998, 13: 132-147.

Guilleminault, C., Clerk, A., Black, J., Labanowski, M., Pelayo, R. and Claman, D. Nondrug Treatment Trials in Psychophysiologic Insomnia. Arch. Intern. Med., 1995, 155: 838844.

Hamer, M. and Chida, Y. (2009). Physical activity and risk of neurodegenerative disease: a systematic review of prospective evidence. Psychol. Med., 2009, 39: 3-11.

Harvey, A. G. (2002). A cognitive model of insomnia. Behav. Res. Ther., 2002, 40: 869-893.

Herdman, M., Gudex, C., Lloyd, A. et al. Development and preliminary testing of the new five-level version of EQ-5D (EQ-5D-5L). Qual. Life. Res., 2011, 20: 1727-1736.

Herring, M. P., O'Connor, P. J. and Dishman, R. K. The effect of exercise training on anxiety symptoms among patients: a systematic review. Arch. Intern. Med., 2010, 170: 321331.

Irwin, M. R., Olmstead, R. and Motivala, S. J. Improving sleep quality in older adults with moderate sleep complaints: A randomized controlled trial of Tai Chi Chih. Sleep, 2008, 31: 1001-1008.

Jeon, C. Y., Lokken, R. P., Hu, F. B. and Van Dam, R. M. Physical Activity of Moderate Intensity and Risk of Type 2 Diabetes: A systematic review. Diabetes care, 2007, 30: 744-752.

Johns, M. W. A new method for measuring daytime sleepiness: The Epworth sleepiness scale. Sleep, 1991, 14: 540-545. 
King, A. C., Oman, R. F., Brassington, G. S. et al. Moderate-intensity exercise and self-rated quality of sleep in older adults: a randomized controlled trial. JAMA, 1997, 277: 32-37.

King, A. C., Oman, R. F., Brassington, G. S., Bliwise, D. L. and Haskell, W. L. Effects of moderate-intensity exercise on polysomnographic and subjective sleep quality in older adults with mild to moderate sleep complaints. J. Gerontol. A Biol. Sci. Med. Sci., 2008, 63: 997-1004.

Kline, C. E., Sui, X., Hall, M. H. et al. Dose-response effects of exercise training on the subjective sleep quality of postmenopausal women: exploratory analyses of a randomised controlled trial. BMJ open, 2011, 2: e001044.

Krupp, L. B., LaRocca, N. G., Muir-Nash, J. et al. The fatigue severity scale: application to patients with multiple sclerosis and systemic lupus erythematosus. Arch. Neurol., 1989, 46: 1121-1123.

Li, F., Fisher, K. J., Harmer, P., Irbe, D., Tearse, R. G. and Weimer, C. Tai Chi and SelfRated Quality of Sleep and Daytime Sleepiness in Older Adults: A Randomized Controlled Trial. J. Am. Geriatr. Soc., 2004, 52, 892-900.

Morin, C. M., Barlow, D. H. and Dement, W. C. Insomnia: Psychological assessment and management (Treatment manuals for practitioners). The Guilford Press, New York, 1993.

Morin, C. M., Belleville, G., Bélanger, L. and Ivers, H. The insomnia severity index: psychometric indicators to detect insomnia cases and evaluate treatment response. Sleep, 2011, 34: 601-608.

Morin, C. M., Bootzin, R. R., Buysse, D. J., Edinger, J. D., Espie, C. A. and Lichstein, K. L. Psychological and behavioral treatment of insomnia: update of the recent evidence (1998-2004). Sleep, 2006, 29: 1398-1414. 
Orff, H. J., Drummond, S. P. A., Nowakowski, S. and Perlis, M. L. Discrepancy between subjective symptomatology and objective neuropsychological performance in insomnia. Sleep, 2007, 30: 1205-1211.

Rimer, J., Dwan, K., Lawlor, D.A. et al. Exercise for depression (Review). Cochrane Database Syst. Rev., 2012, 11:7.

Reid, K. J., Baron, K. G., Lu, B., Naylor, E., Wolfe, L. and Zee, P. C. Aerobic exercise improves self-reported sleep and quality of life in older adults with insomnia. Sleep med., 2010, 11: 934-940.

Sattelmair, J., Pertman, J., Ding, E. L., Kohl Iii, H. W., Haskell, W. and Lee, I. M. Dose Response Between Physical Activity and Risk of Coronary Heart Disease: A MetaAnalysis. Circulation, 2011, 124: 789-795.

Spielberger, C. D., Gorsuch, R. L., Lushene, R., Vagg, P. R. and Jacobs, G. A. Manual for the State-Trait Anxiety Inventory. Consulting Psychologists Press, Palo Alto, CA, 1983.

Troiano, R. P., Berrigan, D., Dodd, K. W., Mâsse, L. C., Tilert, T. and Mcdowell, M. Physical activity in the United States measured by accelerometer. Med. Sci. Sports. Exerc., 2008, 40: 181-88.

Winzer, B., Whiteman, D., Reeves, M. and Paratz, J. Physical activity and cancer prevention: a systematic review of clinical trials. Cancer Causes Control, 2011, 22: 811-26.

World Health Organisation (WHO). Global recommendations on physical activity for health. 2010

Yang, P.-Y., Ho, K.-H., Chen, H.-C. and Chien, M.-Y. Exercise training improves sleep quality in middle-aged and older adults with sleep problems: a systematic review. $J$. Physiother., 2012, 58: 157-63.

Youngstedt, S. D. Effects of exercise on sleep. Clin. Sports Med., 2005, 24: 355-65. 
Youngstedt, S. D., Kripke, D. F. and Elliott, J. A. Circadian phase-delaying effects of bright light alone and combined with exercise in humans. Am. J. Physiol. Regul. Integr. Comp. Physiol., 2002, 282: R259-66.

Youngstedt, S. D., Perlis, M. L., O'brien, P. M. et al. No association of sleep with total daily physical activity in normal sleepers. Physiol. Behav., 2003, 78: 395-401. 